\title{
STUDY ON MAGNETIZATION TREATMENT AND CUTTING PERFORMANCE OF A NEW-TYPE OF TRIETHANOLAMINE BORATE CUTTING FLUID
}

\author{
Yong Yang ${ }^{*}{ }^{\circledR}$, Guofang Hu ${ }^{1}$, Wanxu Liang ${ }^{1}$, Kang Jin ${ }^{1}$, Li Xiong ${ }^{1}$, Shuo Hou ${ }^{1}$ \\ ${ }^{*} 1$ School of Mechanical and Automotive Engineering, Qingdao University of Technology, 266520 \\ Qingdao, China
}

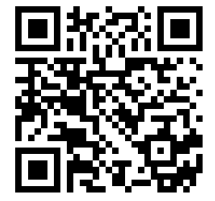

DOI: https://doi.org/10.29121/ijetmr.v7.i11.2020.800

Article Citation: Yong Yang, Guofang Hu, Wanxu Liang, Kang Jin, Li Xiong, and Shuo Hou. (2020). STUDY ON MAGNETIZATION TREATMENT AND CUTTING PERFORMANCE OF A NEW-TYPE OF TRIETHANOLAMINE BORATE CUTTING FLUID. International Journal of Engineering Technologies and Management Research, 7(11), 17-29.

https://doi.org/10.29121/ijetmr.v7 .i11.2020.800

Published Date: 19 November 2020

Keywords:

Triethanolamine Borate Water Based Cutting Fluid Magnetization Treatment

\section{ABSTRACT}

The composition of water-based cutting fluid and emulsion is different, and it has not been reported whether the magnetization treatment will affect the performance of water-based cutting fluid. In this study, a newly developed triethanolamine borate water-based cutting fluid is used as the research object to study the magnetization treatment technology and the law of its influence on cutting performance systematically. The cutting fluid magnetization experimental system was set up based on the principle of magnetization and the influence of magnetization parameters (magnetic field strength, magnetization duration) on the results of magnetization was further studied. The experimental results are measured by force measuring instrument, temperature measuring instrument, roughness measuring instrument and scanning electron microscope, and the parameters such as milling force, milling temperature, surface roughness, cutting specific energy, friction coefficient and workpiece surface morphology are comprehensively analyzed through data processing; the performance of cutting fluid before and after magnetization is also compared. The test results show that the magnetization effect is best when the magnetic field strength is 10000GS and the magnetization time is 40 minutes. The mechanism of magnetization treatment is to change the macromolecular group into a small molecular group, and the disordered array of molecules becomes ordered.

\section{INTRODUCTION}

Titanium alloy has the advantages of high strength and good corrosion resistance and are widely used in aviation industry [1], [2], [3], [4]. However, titanium alloys also have the disadvantages of poor thermal conductivity, low elastic modulus and high chemical activity [5], [6], [7], which makes the processing of titanium alloy very difficult and has always been a problem in the field of aviation manufacturing [8]. Improved cutting fluid is an important means to solve the difficult machining problem of titanium alloy [9], [10]. Cutting fluid can not only form lubricating film between tools, workpieces and chips, but also reduce cutting temperature through gasification and convective heat transfer. In this way, the surface quality of workpiece and tool durability can be improved [11], [12].

At present, many scholars have adopted the method of changing chemical composition of cutting fluid to improve its performance. This method has some limitations and the developed cutting fluid is difficult to adapt to

(C) 2020 The Author(s). This is an open access article distributed under the terms of the Creative Commons Attribution License, which permits unrestricted use, distribution, and reproduction in any medium, provided the original author and source are credited. 
various working conditions. Therefore, it is of great significance to improve the performance of cutting fluid by studying the cutting fluid treatment device. After a long period of research, people think that magnetic field has some mysterious power. So far, magnetic field has been applied in various fields of life, including oil transportation, wastewater treatment, agricultural planting, biomedicine, etc. [13], [14]. The scholars of Harbin University of technology proposed that the particle size of the suspended particles in the emulsion after magnetic field treatment would be smaller, and the magnetization treatment could improve the performance of the emulsion through test verification [15], [16]. Emulsion is a kind of cutting fluid, which contains mineral oil, animal and vegetable oil with a serious waste of resources that can cause pollution to the environment, which lead the green and environmentally friendly water-based synthetic cutting fluid to the development direction of cutting fluid. However, the composition of water-based cutting fluid and emulsion is different. It is necessary to verify whether magnetization can affect the performance of water-based cutting fluid and it will be great significance in the field of metal processing if magnetization can enhance the effect of water-based cutting fluid.

\section{MATERIALS AND METHODS}

\subsection{EXPERIMENTAL MATERIALS}

The tool used in the test is a 68 degree 4-edge tungsten steel milling cutter imported from Switzerland. Its diameter, shank diameter, edge length and overall length are $10 \mathrm{~mm}, 10 \mathrm{~mm}, 25 \mathrm{~mm}$ and $75 \mathrm{~mm}$ respectively. The workpiece used in the test was a TC4 titanium alloy with a density of with density of $4.51 \mathrm{~g} / \mathrm{cm}^{3}$ and the hardness is HRC31. The additive used in the experiment is triethanolamine borate, defoamer is modified polyether and bactericide is isothiazolinone. First, the base liquid is configured, and the base liquid contains $99.5 \%$ Volume deionized water, $0.25 \%$ volume bactericide and $0.25 \%$ volume defoamer. The triethanolamine boric acid ester was mixed with the base fluid according to the volume ratio of 1:10, and then the cutting fluid was prepared and magnetized.

\subsection{EXPERIMENTAL EQUIPMENT AND SCHEME}

The cutting test is carried out on the Wintec MV-80 CNC machining center, with the maximum speed of $5000 \mathrm{r} / \mathrm{min}$. The force measuring instrument used is YDX-III9702 piezoelectric three-way milling force measuring instrument. The thermometer used is thermovision A20 infrared thermal imager. The cooling and lubrication mode of cutting fluid is spray type. The physical figure and schematic diagram of the experimental device are shown in Figure 1. A dynamometer and thermometer are installed before the experiment. The cutting fluid spray device is installed on the machine tool to ensure that the cutting fluid can be sprayed into the contact area during the test; the position and spray volume of the cutting fluid spray device are unchanged during the whole experiment. The processing parameters used in this experiment are spindle speed $1200 \mathrm{r} / \mathrm{min}$, feed speed $200 \mathrm{~mm} / \mathrm{min}$, cutting depth $0.2 \mathrm{~mm}$, cutting width $10 \mathrm{~mm}$. 


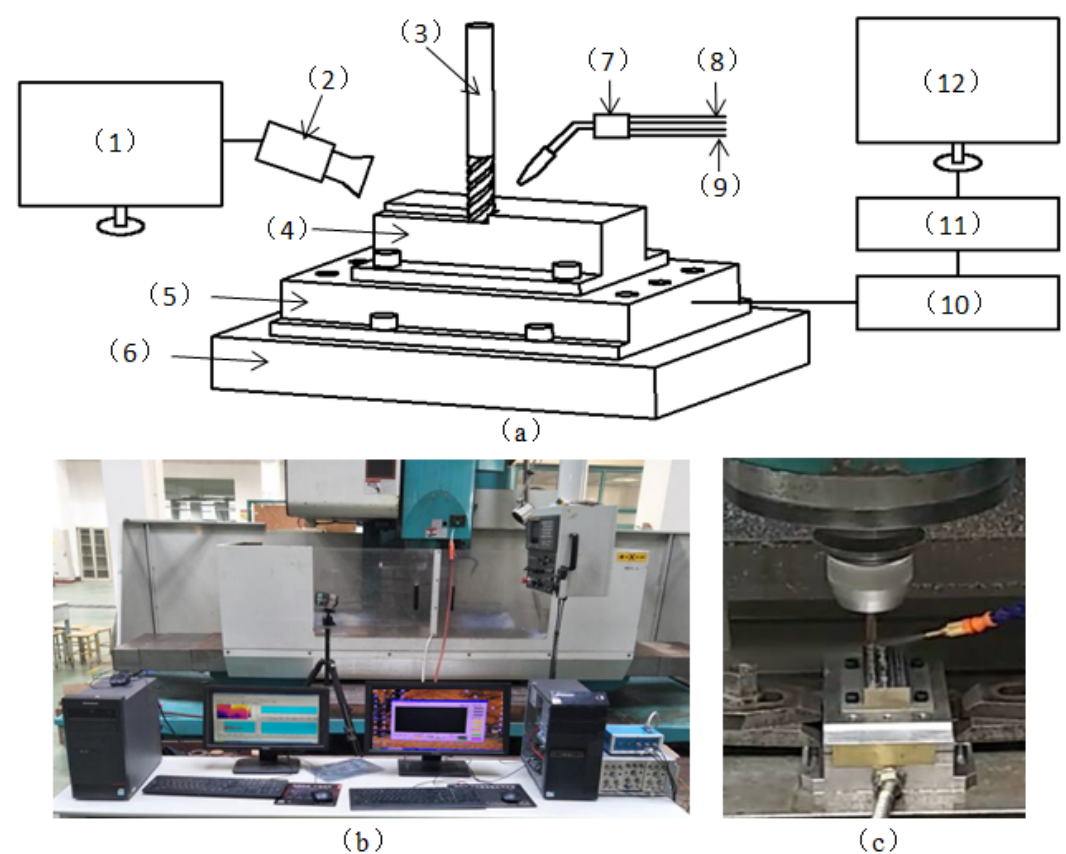

Figure 1: Installation sketch of the milling test. $\mathbf{b}$ Installation physical drawing of the experimental measuring device. c Physical chart of the milling test process. (1) Milling temperature acquisition and analysis software; (2) infrared thermal imager; (3) milling cutter; (4) workpiece; (5) dynamometer; (6) machine tool workbench; (7) atomizer; (8) intake pipe; (9) cutting Fluid Pipe; (10) charge amplifier; (11) data acquisition instrument; (12) milling force acquisition and analysis software.

The magnetization system device of cutting fluid for the test consists of three parts: power cycle, magnetizer and temperature control system. The schematic diagram is shown in Figure 2. The processed cutting fluid then acts on the cutting area through the atomizer. The magnetizer is a permanent magnet type magnetizer, and the structure diagram is shown in Figure 3.

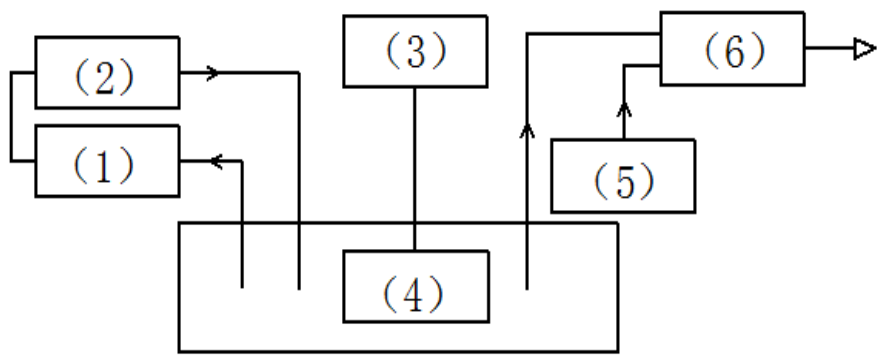

Figure 2: Installation sketch of magnetization system. (1) pump; (2) magnetizer; (3) temperature control system; (4) heater; (5) air compressor; (6) nebulizer.

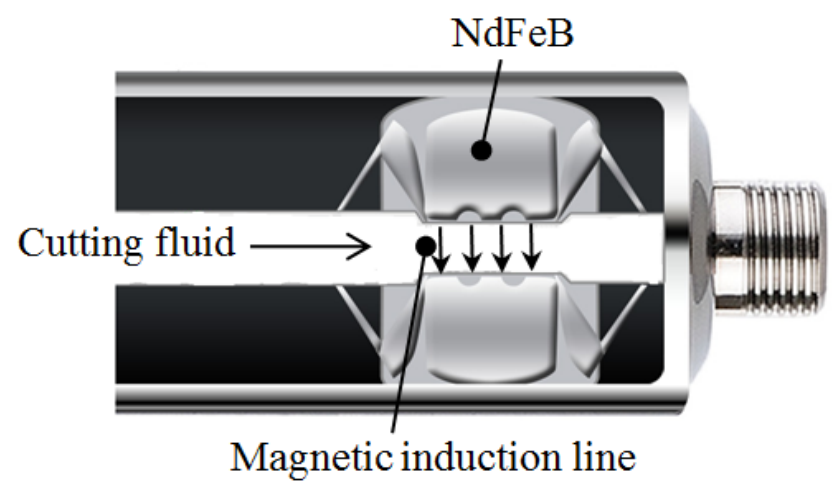

Figure 3: Structural diagram of magnetizer 
The roughness of the machined surface is measured by the TIME3220 roughness meter. The movement direction of the probe is perpendicular to the feed direction of the worktable. After ultrasonic cleaning, S-3400N scanning electron microscope was used to observe the micro morphology of the machined surface.

Firstly, determine the first set of experimental scheme with the magnetization time is 1 hour and magnetizers with magnetic field strengths of about 3000GS and 10000GS are used in experiments to study the influence of magnetic field strength on the magnetization effect. In the second experimental scheme, the magnetic field strength which obtained from the first group of experiments is used to control the magnetic field strength and the cutting fluid temperature is fixed. The magnetization time is changed from 20 minutes to 40 minutes and 60 minutes respectively, and the influence of magnetization time on magnetization effect is studied.

\section{RESULTS AND DISCUSSIONS}

\subsection{ANALYSIS OF THE EFFECT OF MAGNETIC FIELD STRENGTH}

\subsubsection{MILLING FORCE AND TEMPERATURE}

The combined force F is calculated according to the resultant force calculation formula (1) [17].

$$
F=\sqrt{F_{x}{ }^{2}+F_{y}^{2}+F_{z}^{2}}
$$

The milling force and milling temperature are combined to analyze. Figure 4 shows the relationship between the resultant force of cutting fluid and temperature after the treatment of magnetizer with different magnetic field strength. It can be seen from the figure that the combined force decreases with the increase of the magnetic field strength, but the change amplitude is small. However, the milling temperature increases with the increase of magnetic field strength. The milling temperature of $3000 \mathrm{gs}$ and $10000 \mathrm{gs}$ is $42^{\circ} \mathrm{C}$, which is $2^{\circ} \mathrm{C}$ higher than that without magnetization. It is analyzed that this phenomenon is caused by the heat generated by the motor during the magnetization process being absorbed by the cutting fluid. The result of the combined force shows that the magnetizer corresponding to the $10000 \mathrm{gs}$ magnetizer has the lowest combined force, which has a greater impact on the performance of the cutting fluid.

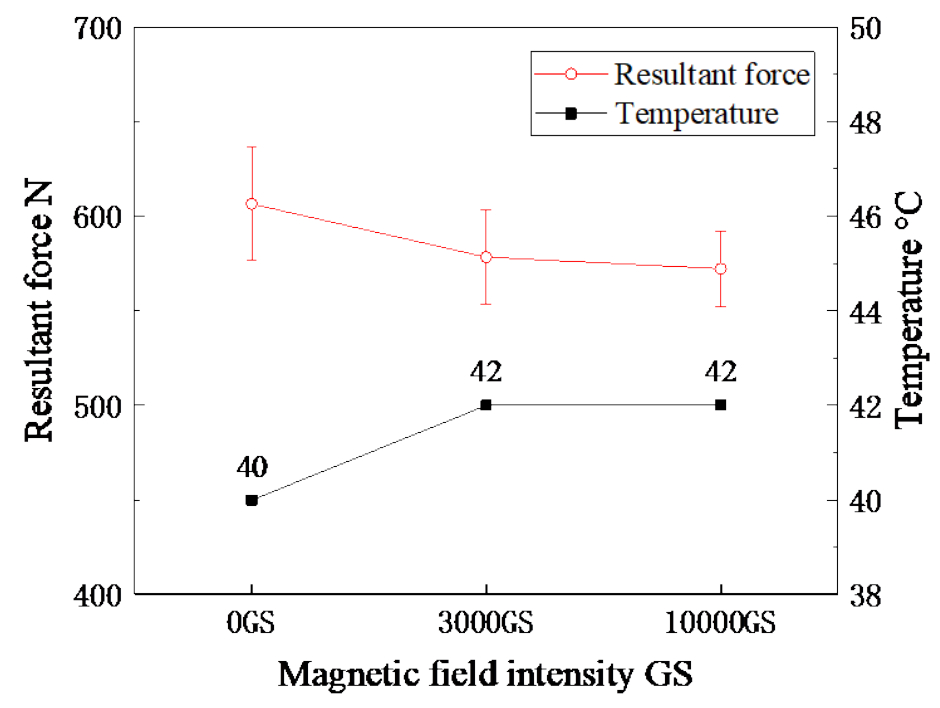

Figure 4: Combined Force and Temperature of Different Magnetic Field Intensities

\subsubsection{SPECIFIC ENERGY OF MILLING}

The specific cutting energy is used to represent the machining efficiency, which is defined as the energy consumed to remove the material per unit volume. The ratio of the cutting energy consumption to the material 
removal volume is obtained. The smaller the specific cutting energy is, the higher the machining efficiency has. The calculation method of milling specific energy is shown in formula (2) [18]. $U$ is the specific energy of cutting $\left(\mathrm{J} / \mathrm{mm}^{3}\right)$, $P$ is the energy consumed (J), $Q_{w}$ is the volume of workpiece removal. $a_{p}$ is the axial depth, $a_{e}$ is the radial depth.

$$
U=\frac{P}{Q_{w}}=\frac{F}{a_{p} \cdot a_{e}}
$$

As shown in Figure 5, the value of specific energy under different magnetic field intensities gradually decreases, which indicates that the processing efficiency increases with the increase of magnetic field intensity, and the specific energy corresponding to $3000 \mathrm{gs}$ and $10000 \mathrm{gs}$ has little difference, but is significantly lower than that corresponding to 0gs. This shows that the efficiency of magnetized cutting fluid is higher than that of unmagnetized cutting fluid, but the specific energy of magnetized cutting fluid is not affected by the magnetic field strength.

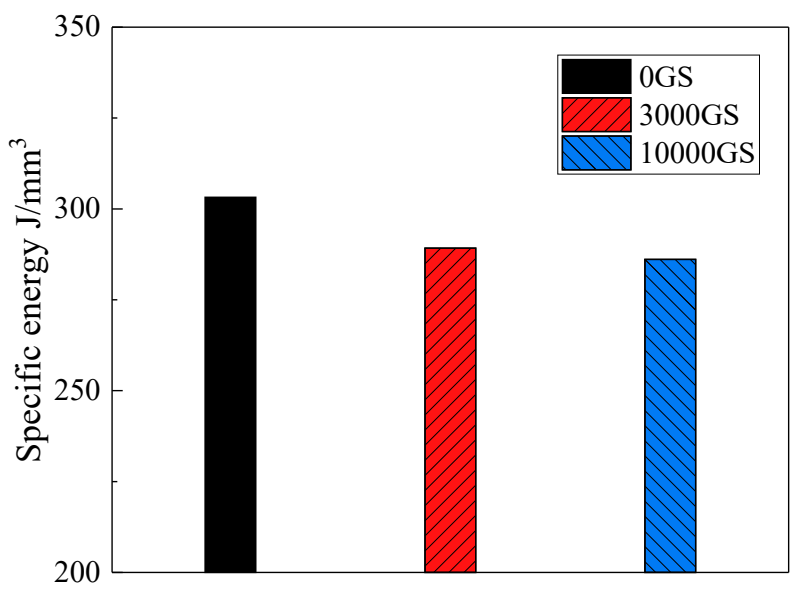

Figure 5: Cutting Specific Energy of Different Magnetic Field Intensity

\subsubsection{FRICTION COEFFICIENT}

The friction coefficient can reflect the lubrication ability of cutting fluid. The smaller the friction coefficient is, the better the lubrication effect of cutting fluid has. The composite cutting force model mentioned by Milton C. Shaw [19] in 《Metal Cutting Principle》 is widely used in the analysis of cutting force, as shown in Figure 6.

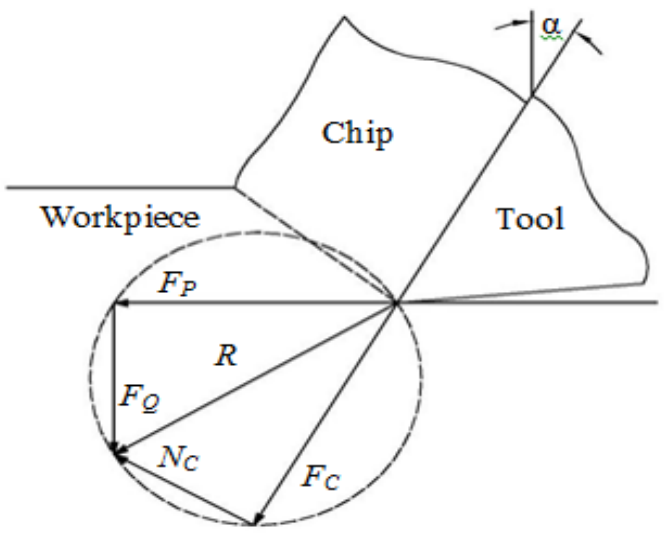

Figure 6: The relationship between milling forces

In Figure $6, F_{C}$ is the force along the tool surface, $N_{C}$ is the force perpendicular to the tool surface, $F_{P}$ is the force in the horizontal direction, $F_{Q}$ is the force in the vertical direction, and $R$ is the combined force of $F_{C}$ and $N_{C}$. The formula for calculating the knife chip interface friction coefficient according to the force shown in the figure is as follows: 


$$
\begin{aligned}
& F_{C}=F_{P} \sin \alpha+F_{Q} \cos \alpha \\
& N_{C}=F_{P} \cos \alpha-F_{Q} \sin \alpha \\
& \mu=\frac{F_{C}}{N_{C}}=\frac{F_{P} \sin \alpha+F_{Q} \cos \alpha}{F_{P} \cos \alpha-F_{Q} \sin \alpha}=\frac{F_{Q}+F_{P} \tan \alpha}{F_{P}-F_{Q} \tan \alpha}
\end{aligned}
$$

According to formula (5), the force measured by the force measuring instrument in $\mathrm{Z}$ direction is brought into force $F_{Q}$, and the arithmetic square root of the sum of squares of forces in X and Y direction is brought into force $F_{P}$ to calculate the friction coefficient.

The friction coefficient corresponding to the cutting fluid treated under different magnetic field strengths is calculated, and the calculation result is shown in Figure 7. It can be seen from the figure that the friction coefficient of untreated cutting fluid is the largest, that cutting fluid treated by 3000GS magnetizer is the second, and that treated by 10000 GS magnetizer is the lowest. Therefore, the lubrication ability of cutting fluid is improved after being treated by magnetizer. Among the magnetizers with different magnetic field strength studied in this experiment, the magnetizer with magnetic field strength of 10000GS has the best effect.

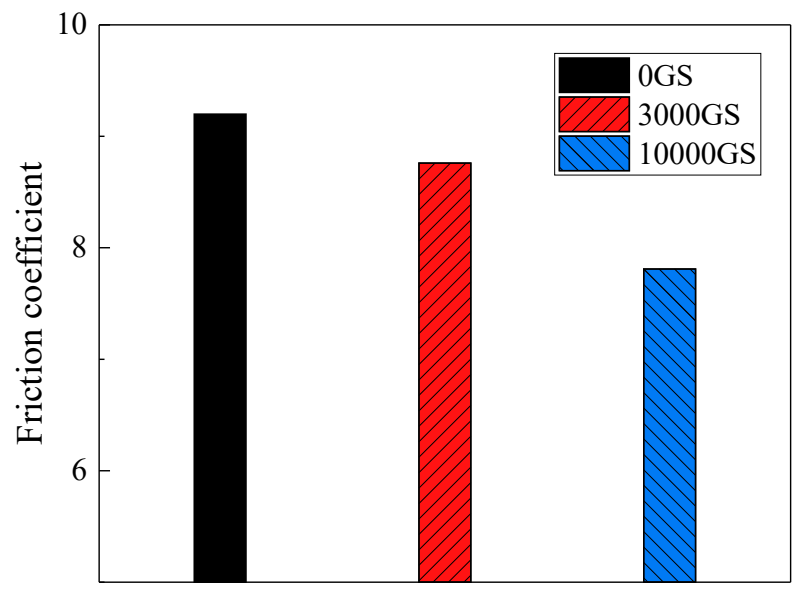

Figure 7: Friction Coefficient of Different Magnetic Field Intensities

\subsubsection{ROUGHNESS}

The roughness of the machined surface is measured by the roughness meter, and the $R a$ and $R S m$ value are taken from the measured roughness signal for analysis. The $R a$ value is the average deviation of the contour arithmetic represented by the height characteristic parameter. The smaller its value is, the smoother the measured surface has. $R S m$ value is the characteristic parameter of spacing, which represents the average length of contour peak and contour valley on the middle line. According to the research of Guo [20], the roughness $R a$ value or $R S m$ value alone cannot fully represent the quality of the machined surface. In the roughness parameters, the weight of $R a$ value is $76 \%$, and the weight of $R S m$ value is $24 \%$. Combined with these two parameters, the comprehensive roughness value is calculated, which is more comprehensive than single factor analysis.

Figure 8 shows the workpiece surface roughness corresponding to the cutting fluid treated with different magnetic field strength. The Ra value increases slightly with the increase of the magnetic field strength, but the variation range is not large, and the RSM value decreases gradually with the increase of the magnetic field strength. According to the comprehensive roughness value obtained from the analysis of the proportion of the two, the influence of the magnetization treatment on the surface roughness of the workpiece is very small. 


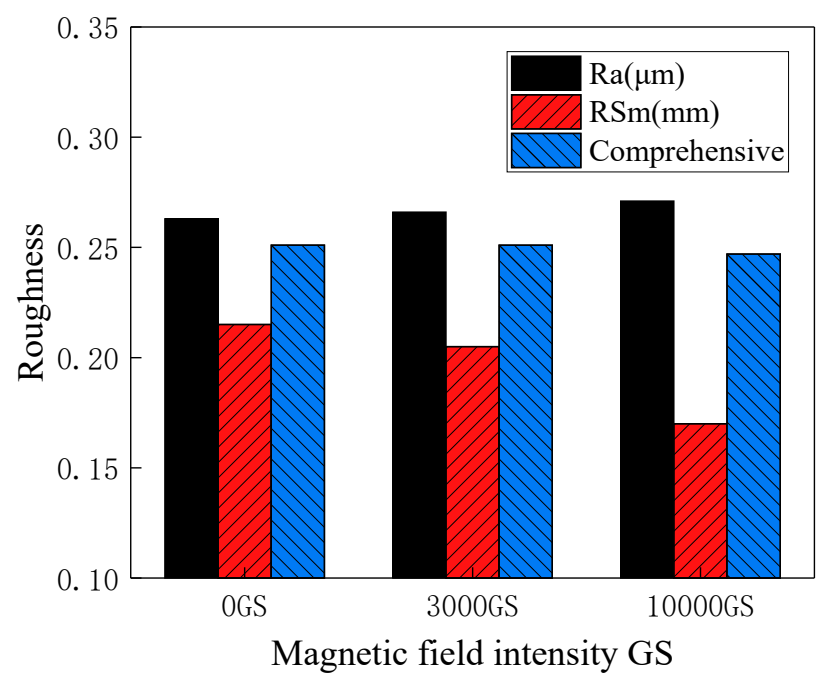

Figure 8: Surface Roughness of Workpiece of Different Magnetic Field Intensity

\subsubsection{SURFACE TOPOGRAPHY}

The scanning electron microscope was used to observe the surface morphology of the workpiece at 100 times magnification, as shown in Figure 9. In the graph of cutting fluid without magnetization treatment, there are individual peeling phenomenon and disordered scratches, while in the graph of magnetic field strength of 3000GS, the scratches become fine and regular, but the peeling characteristics increase significantly, and when the magnetic field strength is $10000 \mathrm{GS}$, the peeling characteristics decrease, at the same time, disordered scratches appear again, and the regular scratches become obvious.

It can be seen that the surface morphology of the workpiece corresponding to the magnetized cutting fluid is higher than that of the non-magnetized cutting fluid, but the corresponding morphologies of the cutting fluid after low magnetic field strength and high magnetic field intensity are different. There are advantages and disadvantages.
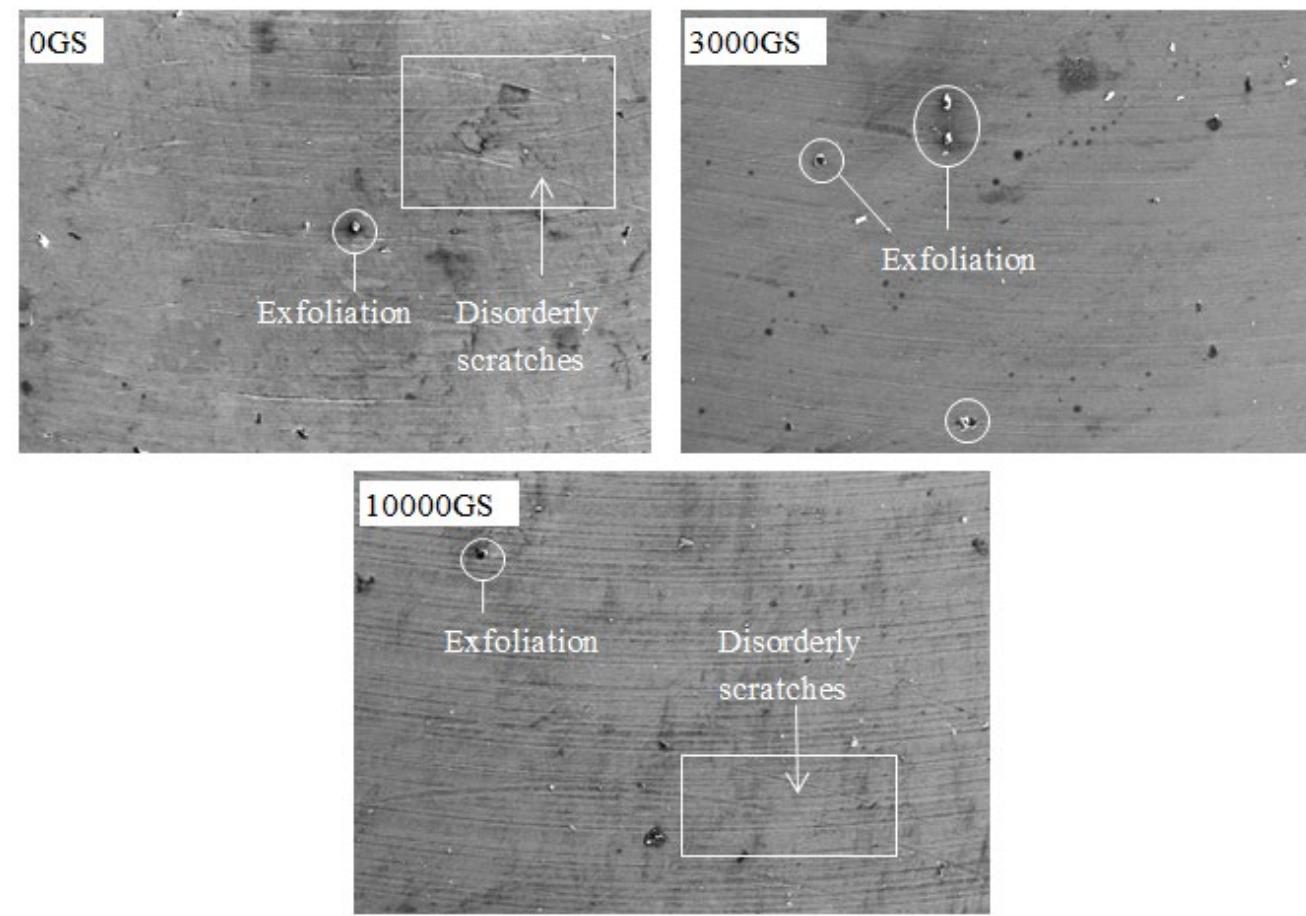

Figure 9: Surface Morphology of Workpiece of Different Magnetic Field Intensity 


\subsection{ANALYSIS OF THE EFFECT OF DURATION OF MAGNETIZATION TREATMENT}

Through the test of changing the magnetic field strength, the parameters of combined force, temperature, specific energy, friction coefficient, roughness and topography are integrated and analyzed. It is found that magnetization treatment has obvious influence on resultant force and friction coefficient, but little influence on roughness. Considering the above parameters, the magnetizer with the magnetic field strength of 10000GS has the greatest influence on improving the performance of cutting fluid. Then taken the magnetizer with the magnetic field strength of $10000 \mathrm{GS}$ as the research object, change the magnetization time, and study the relationship between the performance of cutting fluid and the magnetization time.

\subsubsection{MILLING FORCE AND TEMPERATURE}

Figure 10 shows the variation of milling combined force and milling temperature under the magnetizer with magnetic field strength of 10000GS for different magnetization period. It can be seen from the figure that the resultant force at 20 minutes is significantly lower than that at unmagnetized time, but the change of resultant force after 20 minutes is not obvious. However, the temperature after magnetization is generally higher than that without magnetization, especially reaching the highest value in 40 minutes. This shows that the longer the magnetization time is, the better the effect has, and the longer magnetization time is not applicable in the actual application process, so it is necessary to further analyze and select the cost-effective magnetization time.

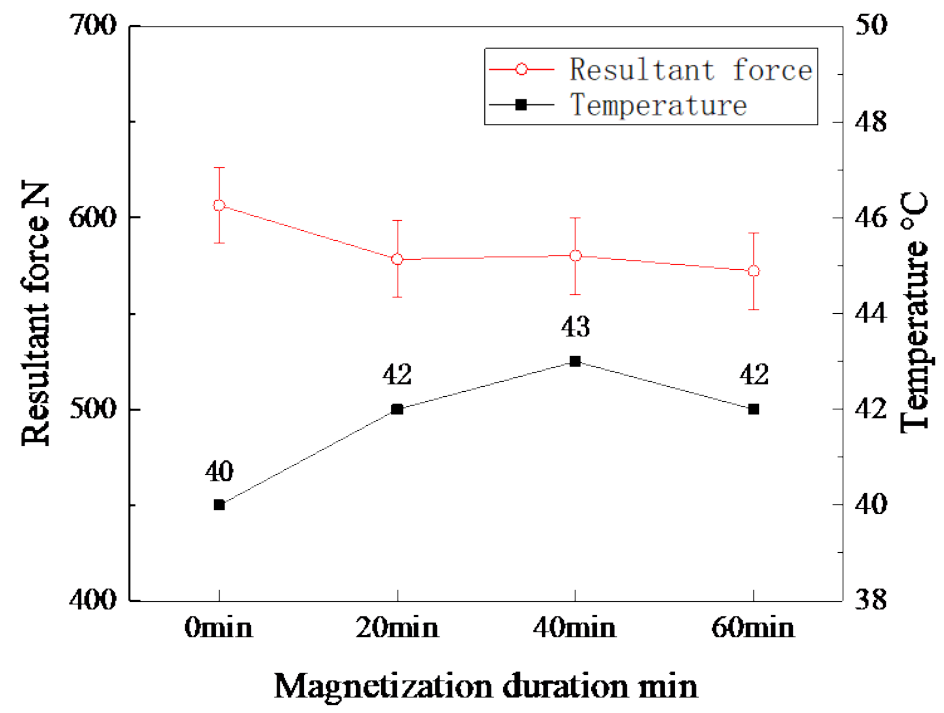

Figure 10: Resultant force and temperature of different magnetization time

\subsubsection{SPECIFIC ENERGY OF MILLING}

The milling specific energy can reflect the machining efficiency of the cutting condition. The milling specific energy corresponding to the different magnetization time length of the cutting fluid is calculated and counted into Figure 11. It can be seen from the figure that the milling specific energy corresponding to the cutting fluid without magnetization treatment is quite different from that corresponding to the cutting fluid after magnetization treatment. Among the magnetized cutting fluid, the milling specific energy is the smallest when the magnetization time is 60 minutes, that is to say, the machining efficiency is the highest. It can be seen that after a short period of magnetization treatment, the effect of cutting fluid will change significantly, but after further enhancing the magnetization time of cutting fluid, the effect will not change too much. 


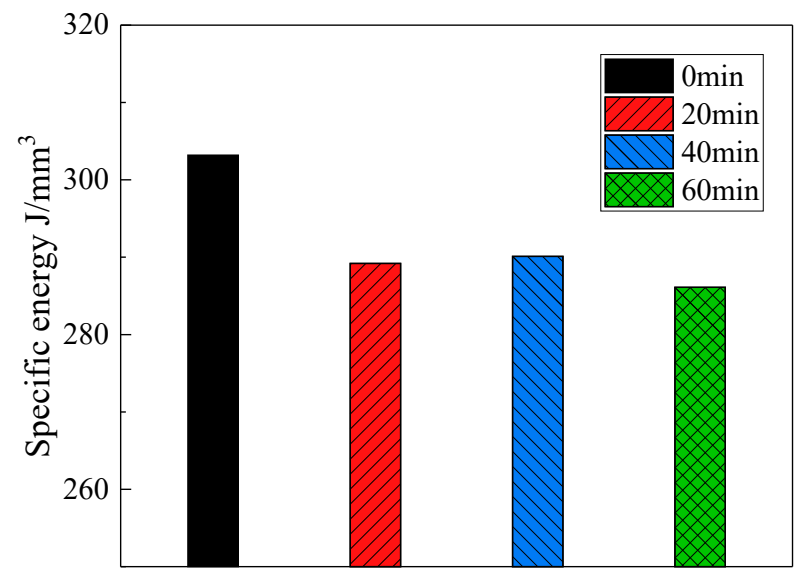

Figure 11: Milling Specific Energy of Different Magnetization Time

\subsubsection{FRICTION COEFFICIENT}

The size of the friction coefficient is the most intuitive embodiment of the strength of the lubrication effect. Figure 12 shows the corresponding friction coefficient of the cutting fluid under different magnetization duration. It can be seen from the figure that the friction coefficient becomes smaller and smaller with the increase of magnetization time, in which the friction coefficient changes greatly when the magnetization time is 20 minutes and 40 minutes, and the difference between the friction coefficient when the magnetization time is 40 minutes and 60 minutes is very small, so it can be inferred that the magnetization effect of cutting fluid can be exerted to a large extent after the magnetization treatment for 40 minutes. After further enhancing the magnetization time of cutting fluid, the effect will not change too much.

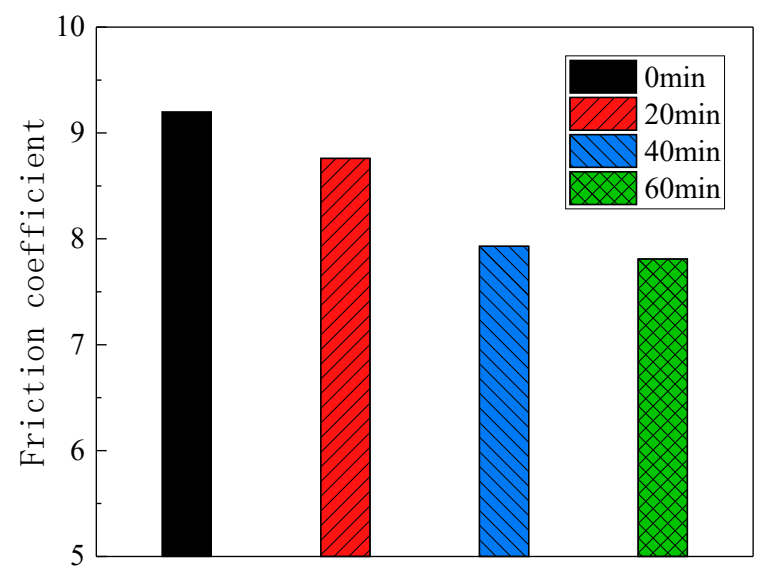

Figure 12: Friction Coefficient of Different Magnetization Time

\subsubsection{ROUGHNESS}

The surface roughness of the workpiece under different magnetization time is measured by the roughness measuring instrument, and the measured roughness $R a$ value, roughness $R S m$ value and roughness comprehensive value are counted into Figure 13 . The $R a$ value and $R S m$ value respectively reflect the surface quality of the workpiece from different aspects. The comprehensive roughness value is calculated according to the weight of a value and $R S m$ value. The following focuses on the comprehensive roughness value. It can be seen from the figure that the comprehensive roughness value decreases gradually 40 minutes before the magnetization treatment, but increases again when the magnetization treatment time is 60 minutes, which is similar to that without magnetization treatment. On the whole, the variation range of the comprehensive roughness value is not large, but the figure shows 
that when the magnetization time is 40 minutes, the comprehensive roughness value is the smallest, and the corresponding cutting fluid has better wear resistance effect.

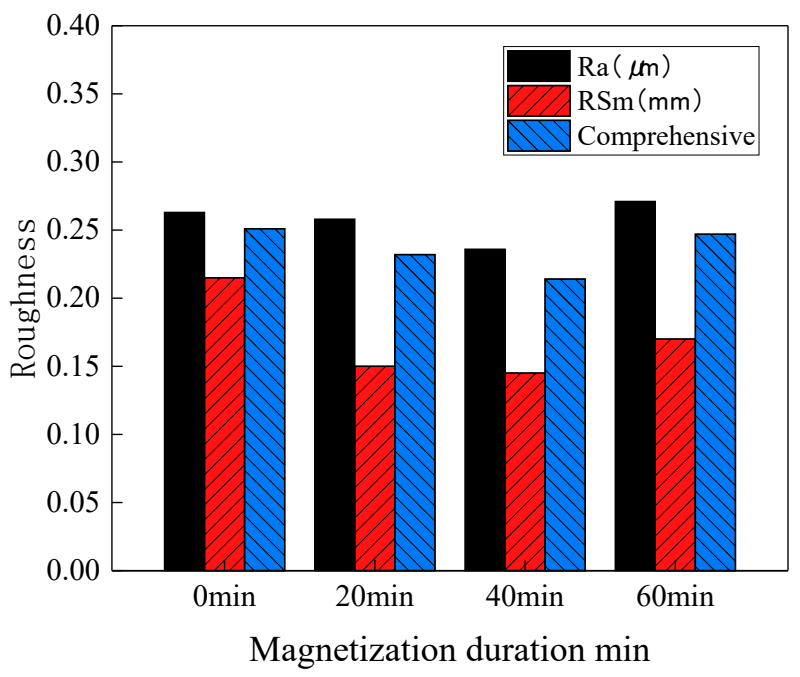

Figure 13: Roughness of Different Magnetization Time

\subsubsection{SURFACE TOPOGRAPHY}

Observe the surface morphology of the workpiece at 100 times of magnification, and draw the surface morphology of the workpiece corresponding to the magnetization time of different cutting fluids into Figure 14. It can be seen from the figure that there are disordered scratches in the surface morphology of the workpiece corresponding to the magnetization time of 0 minutes, 20 minutes and 60 minutes, while there are no messy scratches on the surface morphology of the workpiece corresponding to the magnetization time of 40 minutes, but there are obvious peeling features in the morphology. It is difficult to distinguish which magnetization time is the highest when only considering the surface morphology of the workpiece. If the magnetization time is 40 minutes, the workpiece surface quality is the best, and the corresponding cutting fluid is easier to enter the tool workpiece contact area and play important role of friction reduction and wear resistance.
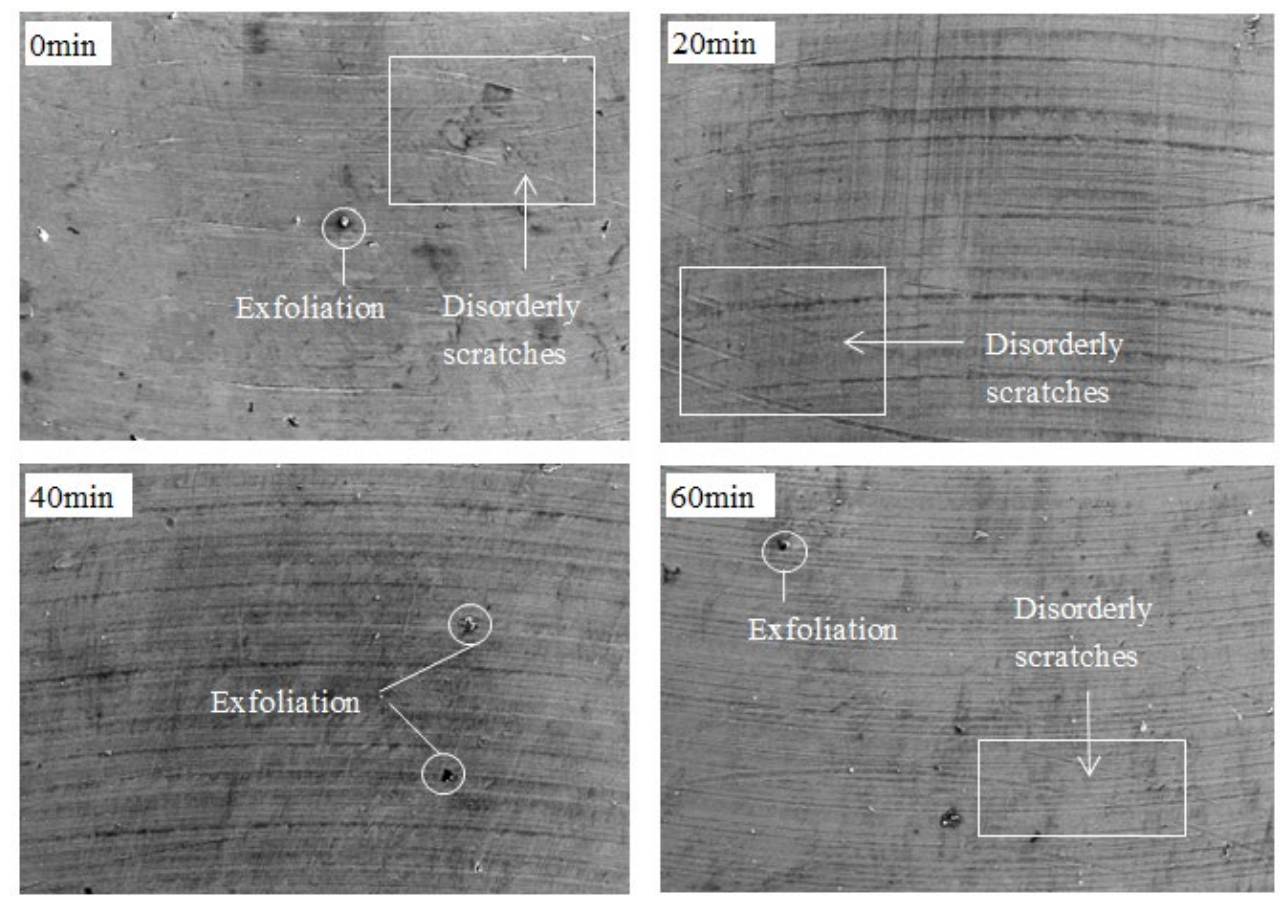

Figure 14: Surface Morphology of Workpiece of Different Magnetization Time 


\subsection{ANALYSIS OF MAGNETIZATION MECHANISM}

By analyzing the effect of magnetic field strength on magnetization, conclusion can be drawn that when the magnetic field strength is $10000 \mathrm{GS}$, the effect of magnetized cutting fluid is the best, and the reason why cutting fluid can improve its performance after passing through the magnetic field may be that the molecular group of cutting fluid becomes smaller, and the ions in the solution are orderly arranged under the effect of magnetic field. The schematic diagram of the action process is shown in Figure 15.

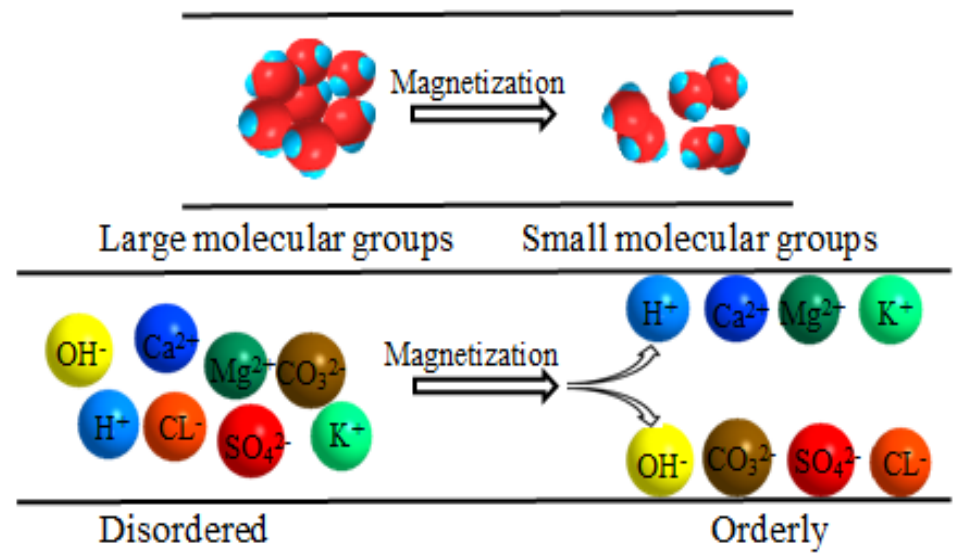

Figure 15: Schematic diagram of magnetization process

In the micro case, when the positive and negative particles in the cutting fluid pass through the magnetic field, their existence mode changes from disorderly distribution to orderly arrangement. Under the action of the magnetic force, all kinds of particles move at the same time and collide which causes the hydrogen bond between the molecules trends to break, and makes the original large sub cluster become small molecular cluster which is easier to penetrate into the cutting tools, workpieces and chips during the milling process. In the contact area, a temporary boundary lubrication film is formed and play an important role in the friction and wear reducing, as shown in Figure 16. At present, this assumption only belongs to the theoretical inference This experiment studies are restricted by existing equipment in detecting the molecular arrangement and particle size. It can only be proved indirectly using the results of milling test.

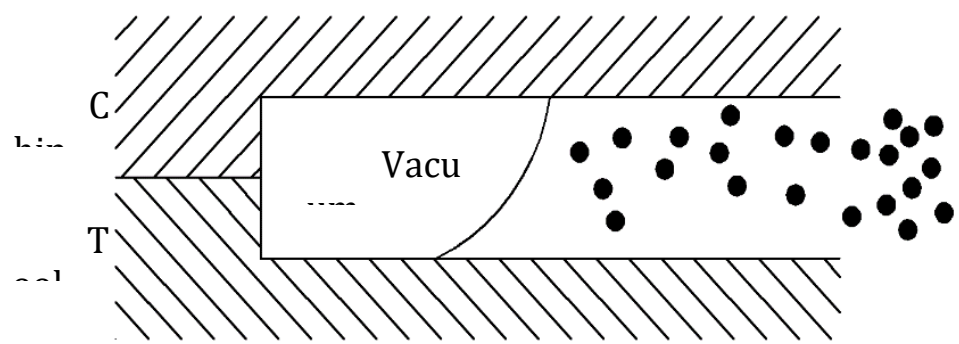

(a) Disordered infiltration before magnetization

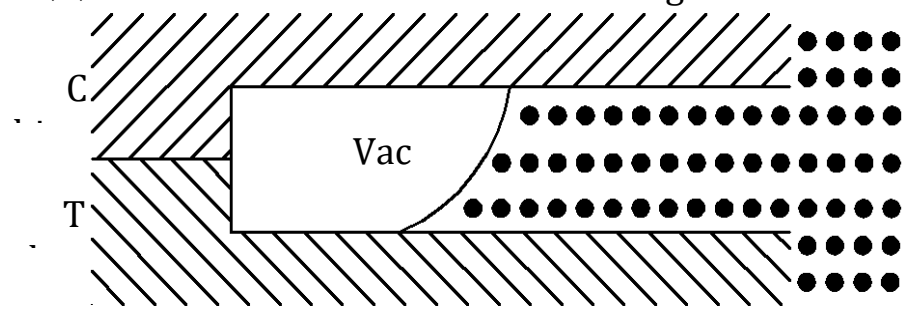

(b) Orderly infiltration after magnetization

Figure 16: molecular action state of cutting fluid before and after magnetization 


\section{CONCLUSIONS \& RECOMMENDATIONS}

1) The results show that the magnetic field strength of 10000GS is better than that of 3000GS and 0GS in the three aspects of resultant force, specific energy and friction coefficient. This shows that magnetization treatment can have a favorable effect on water-based total synthetic cutting fluid, and the magnetization effect is related to the magnetic field strength. Among the three magnetic field strengths studied in this paper, 10000GS has the best magnetization effect.

2) By analyzing the parameters of friction coefficient, roughness and workpiece surface morphology, it can be found that the magnetization time has a great influence on the magnetization effect in a short period of time, and the magnetization time increases after 40 minutes has a little influence on the magnetization effect. Among the four magnetization durations studied in this paper, when the magnetization duration is 40 minutes, the cost performance of magnetization treatment is the highest.

3) Experiments shows that the performance of the magnetized cutting fluid is better, so the mechanism of the magnetization process is deduced theoretically: the macromolecule group is changed into the micromolecule group by the effect of magnetic force, and the molecules are arranged orderly, which makes the cutting fluid molecules easier to enter the cutting area to exert the lubricating effect.

\section{SOURCES OF FUNDING}

This research received no specific grant from any funding agency in the public, commercial, or not-for-profit sectors.

\section{CONFLICT OF INTEREST}

The author have declared that no competing interests exist.

\section{ACKNOWLEDGMENT}

This research was financially supported by the following Foundation items: Shandong Provincial Natural Science Foundation, China (ZR2017MEE077).

\section{REFERENCES}

[1] Eguti C C A, Trabasso L G. Design of a robotic orbital driller for assembling aircraft structures[J]. Mechatronics, 2014, 24(5):533-545.

[2] Denkena B, Boehnke D, Dege J H. Helical milling of CFRP-titanium layer compounds[J]. CIRP Journal of Manufacturing Science and Technology, 2008, 1(2):64-69.

[3] Dorlin, Theo, Fromentin G, Costes J P. Generalised cutting force model including contact radius effect for turning operations on Ti6Al4V titanium alloy[J]. The International Journal of Advanced Manufacturing Technology, 2016.

[4] Bui V H, Gilles P, Sultan T, et al. A new cutting depth model with rapid calibration in abrasive water jet machining of titanium alloy[J]. The International Journal of Advanced Manufacturing Technology, 2017.

[5] Yang Y, Zhang C, Dai Y, et al. Lubricity and Adsorption of Castor Oil Sulfated Sodium Salt Emulsion Solution on Titanium Alloy[J]. Tribology Letters, 2019.

[6] Zhao D. Development and application of titanium alloy in aviation field [J]. Foundry, 2014,63 (11): 1114-1117

[7] Liu J. Characteristics and application of titanium alloy [J]. Non ferrous metal processing, 2002,31 (04): 1-9 + 59

[8] Ezugwu E O. Key improvements in the machining of difficult-to-cut aerospace superalloys[J]. International Journal of Machine Tools \& Manufacture, 2005, 45(12-13):1353-1367.

[9] E. Brinksmeier, D. Meyer, A.G. Huesmann-Cordes, et al. Metalworking fluids-Mechanisms and performance[J]. CIRP Annals - Manufacturing Technology, 2015. 
[10] Osama M, Singh A, Walvekar R, et al. Recent developments and performance review of metal working fluids[J]. Tribology International, 2017, 114:389-401.

[11] Xavior M A, Adithan M. Determining the influence of cutting fluids on tool wear and surface roughness during turning of AISI 304 austenitic stainless steel[J]. Journal of Materials Processing Technology, 2009, 209(2):900909.

[12] Khandekar S, Sankar M R, Agnihotri V, et al. Nano-Cutting Fluid for Enhancement of Metal Cutting Performance[J]. Materials and Manufacturing Processes, 2012, 27(9):963-967.

[13] Yan T, Zhang Y, Yan S, Quan Y. Mechanism analysis of magnetic field in wastewater treatment [J]. Science and technology innovation, 2019 (26): 57-58

[14] Li W. Study on the influence of ultrasonic, magnetic and electric field treatment on the germination vigor of soybean seeds [J]. Agricultural research and application, 2017 (06): 21-25

[15] Chen X. Experimental study on the cooling and lubricating properties of magnetized emulsion [D]. Harbin University of technology, 2008

[16] Li X. Influence of blade shape and emulsion treatment on the machinability of GH4169 [D]. Harbin University of technology, 2011

[17] Yin Q, Li C, Zhang Y, et al. Spectral analysis and power spectral density evaluation in Al2O3 nanofluid minimum quantity lubrication milling of 45 steel [J]. The International Journal of Advanced Manufacturing Technology. 2018, 97, 129-145.

[18] Zhao M. Basic research on size effect of micro milling [D]. Nanjing University of Aeronautics and Astronautics, 2014.

[19] Shaw M C.Metal Cutting Principles [M]. second Edition. Oxford University Press,2005.

[20] Guo S, Li C, Zhang Y, et al. Analysis of volume ratio of castor/soybean oil mixture on minimum quantity lubrication grinding performance and microstructure evaluation by fractal dimension[J]. Industrial Crops and Products, 2018, 111:494-505. 